\title{
Intravitreal bevacizumab for the treatment of macular oedema secondary to branch retinal vein occlusion
}

\author{
T C Kreutzer, ${ }^{1}$ C S Alge, ${ }^{1}$ A H Wolf, ${ }^{1}$ D Kook, ${ }^{1}$ J Burger, ${ }^{1}$ R Strauss, ${ }^{1}$ C Kunze, ${ }^{2}$ \\ C Haritoglou, ${ }^{1}$ A Kampik, ${ }^{1}$ S Priglinger ${ }^{1}$
}

${ }^{1}$ Department of Ophthalmology, Ludwig-Maximilians-University, Munich, Germany; ${ }^{2}$ Department of Ophthalmology, Medical University, Innsbruck, Austria

Correspondence to:

Dr S G Priglinger, Department of Ophthalmology, LudwigMaximilians-University, Munich, Mathildenstr 8, 80336 Munich, Germany; siegfried.priglinger@ med.uni-muenchen.de

Accepted 27 November 2007 Published Online First 22 January 2008

\section{ABSTRACT}

Purpose: To evaluate the effect of intravitreal bevacizumab (Avastin) injections on visual acuity (VA) and foveal retinal thickness in patients with macular oedema secondary to branch retinal vein occlusion.

Methods: A prospective, non-comparative, consecutive, interventional case series of 34 patients. Patients received repeated intravitreal injections of $1.25 \mathrm{mg}$ bevacizumab. Main outcome measures were VA (Snellen charts and ETDRS) and retinal thickness (optical coherence tomography measurements) in a follow-up period of 6 months. Results: Patients presented at a mean age of 69 years (range 44-86). Mean duration of symptoms was 40 weeks (range 1-300). Mean (SD) VA at baseline was 0.79 (0.39) logMAR, improving to 0.51 (0.34) logMAR at 6 months $(p=0.009)$. Mean number of letters on the ETDRS chart at baseline was 45.3 (19.0), improving to $60.6(19.9)$ at 6 months $(p=0.003)$. Mean (SD) retinal thickness at baseline was 474 (120) $\mu \mathrm{m}$, declining to 316 (41) $\mu \mathrm{m}$ at 6 months.

Conclusion: Intravitreal injection of $1.25 \mathrm{mg}$ bevacizumb appears to be an effective treatment option for branch retinal vein occlusion.

Branch retinal vein occlusion (BRVO) is a common retinal vascular disorder of the elderly.

Associated risk factors include advanced age (usually 60-70 years), arterial hypertension, arteriolosclerosis, diabetes mellitus, hyperlipidaemia and smoking. ${ }^{1}$ Visual acuity is usually affected only when the area of ischaemia includes the macula or if secondary oedema and/or bleeding involve the macula. The only evidence-based treatment available for macular oedema secondary to BRVO is retinal grid laser photocoagulation based on the results of the Branch Vein Occlusion Study. ${ }^{2}$ However, visual outcome for laser-treated patients in this study was a mean gain of 1.33 lines, suggesting the need for advanced therapeutic measurements. In addition, one major drawback of laser photocoagulation is its after-effect of visual field defects, which my increase over time.

Recent studies have shown vascular endothelial growth factor (VEGF) to play a crucial role in the pathogenesis of retinal oedema secondary to vasoocclusive disease. ${ }^{3-5}$ VEGF has been reported to destabilise endothelial tight junctions and promote endothelial cell proliferation secondary to ischaemia, both conditions found in vaso-occlusive retinal disease. ${ }^{67}$ Upregulation of VEGF is associated with the breakdown of the blood/retina barrier, with the increased vascular permeability resulting in retinal oedema, stimulation of endothelial cell growth, and neovascularisation. ${ }^{8-10}$
Thus pharmacological inhibition of VEGF appears to be a promising approach for treatment of BRVO, in which the breakdown of the blood/ retinal barrier and neovascularisation have important roles.

The VEGF inhibitor bevacizumab (Avastin, rhuMAb-VEGF; Genentech, South San Francisco, California, USA) is a full-length humanised monoclonal antibody approved by the Food and Drug Administration, which was originally developed for the treatment of metastatic colorectal cancer. It has recently emerged as a novel therapeutic strategy for retinal diseases, especially age-related macular degeneration, and, in retrospective shortterm studies, has also proven to be effective in central retinal and branch retinal vein occlusion. ${ }^{511}$ None of the clinical and experimental studies published so far have found any drug-related toxic effects on any retinal structures.

This study was conducted to prospectively evaluate the effect of intravitreal administration of $1.25 \mathrm{mg}$ bevacizumab on macular oedema and visual function secondary to BRVO.

\section{METHODS}

\section{Study design}

This study was designed as a prospective, consecutive, non-comparative case series.

Patient eligibility with regard to diagnosis of macular oedema after BRVO was confirmed from optical coherence tomography (OCT) images, fluorescein angiograms and fundus photographs. Patients who had received previous laser treatment within the preceding 6 months were excluded.

All patients gave their written informed consent. They were specifically informed about the off-label character of the treatment and the potential risk of retinal detachment and endophthalmitis, as well as the fact that additional treatment might be required. The study was performed in accordance with the Declaration of Helsinki. Only one eye was selected as the study eye.

In an initial step, patients received an intravitreal injection of $1.25 \mathrm{mg}$ bevacizumab on day 1 and 4 weeks thereafter. After the second injection, the decision on further intravitreal administration of bevacizumab was made on the basis of treatment success, ineffectiveness or toxicity as determined by evaluation of visual acuity and OCT findings. Patients underwent monthly visual acuity testing, ocular pressure measurement, slit lamp and stereoscopic fundus examination, OCT imaging, and fundus photography. 
Owing to the off-label character of the intravitreal bevacizumab treatment, a strict treatment protocol was developed to avoid ineffective treatment.

\section{Treatment success}

Treatment success after the second intravitreal bevacizumab injection was determined as follows: (a) best corrected visual acuity score of the study eye of $\geqslant 79$ letters (approximate Snellen equivalent of $\geqslant 20 / 30)$; $(b)$ average retinal thickness in the OCT central subfield of $\leqslant 225 \mu \mathrm{m}$. If treatment was discontinued because of success, the patient nevertheless continued to undergo the scheduled monthly assessments. Treatment was restarted if the average macular thickness of the study eye increased by $\geqslant 50 \mu \mathrm{m}$, as assessed on OCT scans, or visual acuity decreased by $\geqslant 5$ letters and was $<74$ letters.

\section{Treatment ineffectiveness (ie, not even borderline improvement)}

If two consecutive monthly bevacizumab injections had not produced at least borderline improvement in the study eye, the treatment was discontinued. Borderline improvement was defined as follows: (a) a decrease in mean retinal thickening of the study eye of at least $50 \mu \mathrm{m}$; $(b)$ an increase in best corrected visual acuity score of at least 5 letters. If the study eye did not show borderline improvement, further treatment with bevacizumab injections was discontinued at the 2-month follow-up.

Patients who discontinued treatment for this reason were also enrolled in the scheduled monthly assessments.

\section{Outcome measures}

The main outcome measures of the treatment were central retinal thickness and visual function. Best corrected visual acuity was tested using Snellen-adjusted charts (Moeller-Wedel M3000, Wedel, Germany) projected at $5 \mathrm{~m}$ distance with numeric presentation. Letter numbers were then evaluated using ETDRS charts (Lighthouse International, New York, USA). ${ }^{12}$ Additional assessments, consisting of intraocular pressure measurement, slit lamp examination, bilateral stereoscopic fundus biomicroscopy using a 78 diopter lens, retinal thickness measurement using OCT (Stratus OCT-3000; Carl Zeiss Meditec, Dublin, California, USA), and fundus photographs of the posterior pole and the macular area, were performed before treatment and during the follow-up examinations 14 days, 6 weeks and 3, 4 and 6 months after treatment. For statistical analysis, Snellen acuity was converted into the corresponding value in logarithmic minimal angle of resolution (logMAR).

\section{Injection technique}

Topical anaesthesia was achieved before injection using 1\% tetracaine eye drops. Before injection, the eye was scrubbed with $10 \%$ povidone/iodine. Patients then received a unilateral intravitreal injection of $0.05 \mathrm{ml}$ containing $1.25 \mathrm{mg}$ bevacizu-

Table 1 Visual acuity (logMAR, letters on ETDRS chart and OCT) after treatment with bevacizumab

\begin{tabular}{llllll}
\hline & Baseline & 2 weeks & $\mathbf{6}$ weeks & 3 months & 6 months \\
\hline logMAR & $0.79(0.39)$ & $0.64(0.41)^{*}$ & $0.55(0.35)^{*}$ & $0.49(0.39)^{*}$ & $0.51(0.34)^{*}$ \\
ETDRS & $45.3(19.0)$ & $51.4(20.8)^{*}$ & $58.7(19.8)^{*}$ & $64.1(21.4)^{*}$ & $60.6(19.9)^{*}$ \\
OCT & $474(120)$ & $368(82)^{*}$ & $333(66)^{*}$ & $331(58)^{*}$ & $316(41)^{*}$ \\
\hline
\end{tabular}

Values are mean (SD).

${ }^{*} p<0.05$ compared with baseline value (Wilcoxon test).

logMAR, logarithm of minimal angle of resolution; OCT, optical coherence tomography. mab using a sharp 27-gauge needle at a distance of 3.5-4.0 mm from the limbus.

\section{Statistical analysis}

All data were collected in a MS-Excel 2000 spreadsheet (Microsoft Corporation, Unterschleissheim, Germany) and analysed using SPSS V13.0 for Windows (SPSS Inc, Chicago, Illinois, USA). For statistical analysis, the Wilcoxon test was applied for comparison of multivariables within one group (eg, initial visual acuity versus final visual acuity) and the MannWhitney $U$ test was performed for comparison of differences between subgroups. For all statistical tests, $p<0.05$ was considered significant.

\section{RESULTS}

Thirty-four patients (21 male) with retinal oedema involving the centre of the macula secondary to BRVO were evaluated. Patients presented at a mean age of 69 years (range 44-86). Mean duration of symptoms from BRVO until study inclusion was 40 weeks (range 1-300). All 34 patients completed the 6month observation period, attending all control visits.

Fourteen $(41 \%)$ of the 34 eyes had been pretreated. Two eyes $(6 \%)$ had received a pars plana vitrectomy with peeling of epiretinal membranes, one with and one without additional sheathotomy. Eleven eyes (32\%) had received laser photocoagulation for treatment of macular oedema, three of them combined with intravitreal injection of triamcinolone acetate. One patient $(3 \%)$ had received an intravitreal injection of triamcinolone acetate alone. The remaining 20 (59\%) had not been treated before study inclusion.

The mean number of intravitreal injections performed during the study period was 2.9. Altogether, 15 (44\%) eyes received two, $10(29 \%)$ eyes three, six (18\%) eyes four, two (6\%) eyes five, and one (3\%) eye six injections.

Overall mean (SD) visual acuity at baseline was $0.79(0.39)$ logMAR (20/120), improving to 0.51 (0.34) logMAR (20/60) at 6 months $(p<0.001)$ (table 1$)$. To be more precise, whereas 29 $(85 \%)$ of the 34 eyes included in the study initially had visual acuity worse than 20/40 and only five eyes with 20/40 or better $(15 \%)$, at 6 months, the visual acuity was worse than $20 / 40$ in only 21 cases (62\%) and was 20/40 or better in 13 cases (38\%). At 6 months, the gain in visual acuity was two or more lines in $21(62 \%)$ and four or more lines in $12(35 \%)$ of the 34 cases.

At baseline, the mean number of letters was 45.3 (19.0) and this improved to $60.6(19.9)$ at 6 months $(p<0.001)$. Overall, mean gain between baseline and 6 months was 15.3 (13.5) letters $(p<0.001)$.

Retinal thickness measured by OCT was $474(120) \mu \mathrm{m}$ at baseline and declined to $316(41) \mu \mathrm{m}$ at 6 months $(p<0.001)$.

Subgroup analysis of pretreated cases versus those not pretreated revealed some statistically significant differences. The 14 pretreated eyes showed a mean visual acuity of 0.91 (0.40) logMAR (20/160) at baseline and a mean of 38.0 (17.4) letters, whereas the 20 eyes that had not been pretreated showed a mean baseline visual acuity of $0.71(0.38) \log M A R$ $(20 / 200)$ and a mean of 50.4 (18.9) letters $(p=0.120$ and 0.047 , respectively). At 6 months, eyes that had not been pretreated showed a mean visual acuity of 0.37 (0.27) logMAR (20/50) and a mean of 67.7 (16.5) letters and pretreated eyes achieved a mean visual acuity score of only $0.70(0.36) \log$ MAR (20/200) and a mean of 50.5 (20.6) letters ( $p=0.04$ and 0.012 , respectively) (figs 1 and 2 ). In addition, there was a mean letter 


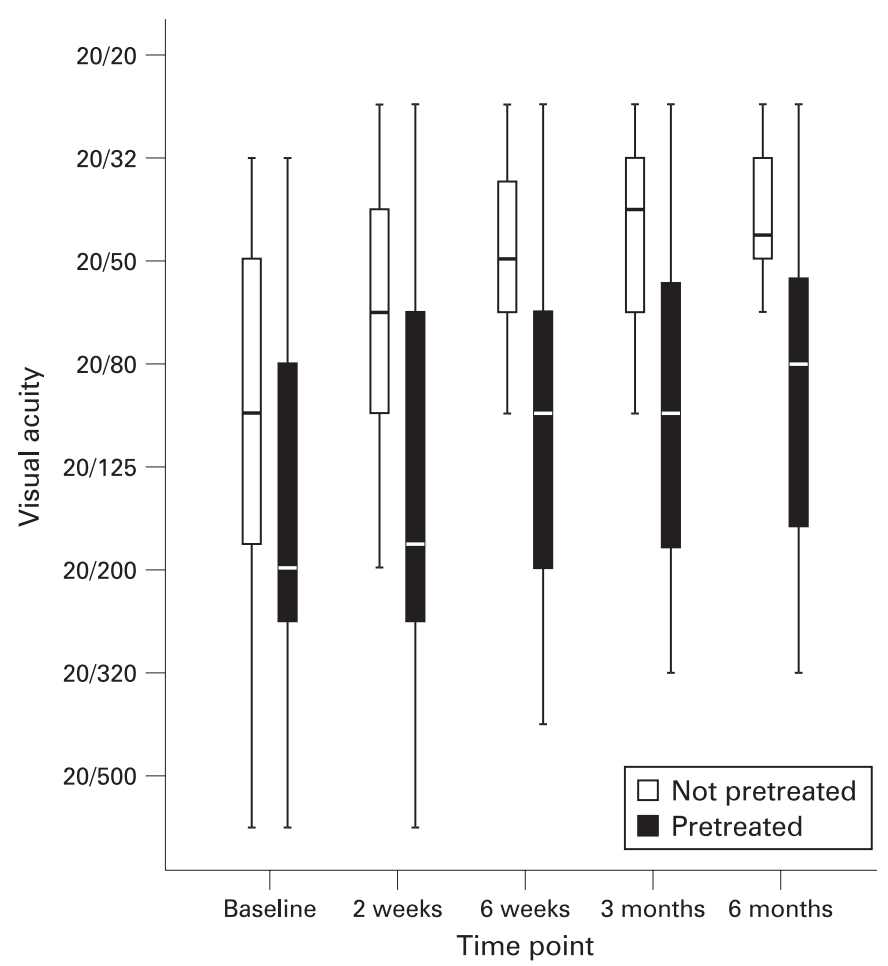

Figure 1 Comparison of visual acuity results between pretreated $(n=14)$ and non-pretreated $(n=20)$ cases of branch retinal vein occlusion.

gain of 17.3 (15.3) for eyes that had not been pretreated compared with only 12.5 (11.3) in pretreated eyes $(p=0.592)$.

Although treatment effects in non-pretreated eyes seemed more pronounced, both pretreated and untreated eyes showed significant improvement, as assessed by both $\log M A R$ visual acuity ( $p=0.012, p=0.004$, respectively) and letter scores $(p<0.001$ for both).

Comparison of retinal thickness measured by OCT between the two groups revealed no significant differences at either baseline or 6 months ( $p=0.478$ and 0.071 , respectively) (fig 3). At baseline, pretreated eyes had a mean central retinal thickness of $492(179) \mu \mathrm{m}$, decreasing to 332 (28) $\mu \mathrm{m}$ after 6 months $(p=0.004)$, and the eyes that had not been pretreated had a baseline retinal thickness of 471 (111) $\mu \mathrm{m}$, which declined to 304 (46) $\mu \mathrm{m}$ at 6 months $(\mathrm{p}<0.001)$.

Further subgroup analysis was performed to evaluate the effect of the duration of symptoms (decrease in visual acuity secondary to BRVO) before initiation of treatment with intravitreal injections of bevacizumab. In 13 (38\%) eyes, treatment was initiated within 90 days of the initial decrease in visual acuity, and $21(69 \%)$ patients were treated more than 90 days after the initial perception of visual decline. Statistical analysis of these two subgroups revealed no significant difference in visual acuity or central retinal thickness (each $\mathrm{p}>0.366$ ).

In five eyes (15\%), minimal recurrent exudation with macula oedema was found at the 5 -month visit. In all these cases, the macula was considered to be dry at the 4-month control visit after three intravitreal injections of bevacizumab. At the 5month visit, these eyes showed new minor exudation with little decrease in visual acuity. After fluorescence angiographic evaluation, additional focal laser photocoagulation was performed. Exudation was successfully eliminated and retinal thickness was stabilised at the 6-month visit.

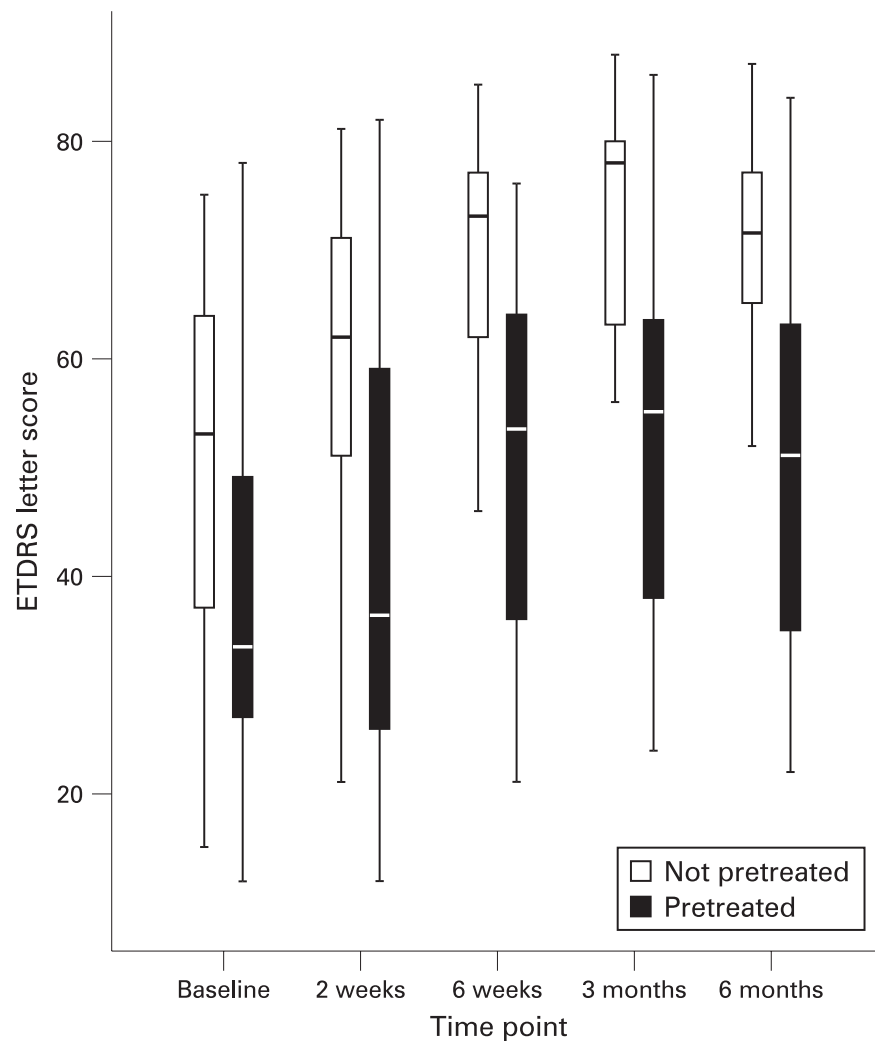

Figure 2 Comparison of ETDRS letter scores between pretreated $(n=14)$ and non-pretreated $(n=20)$ cases of branch retinal vein occlusion.

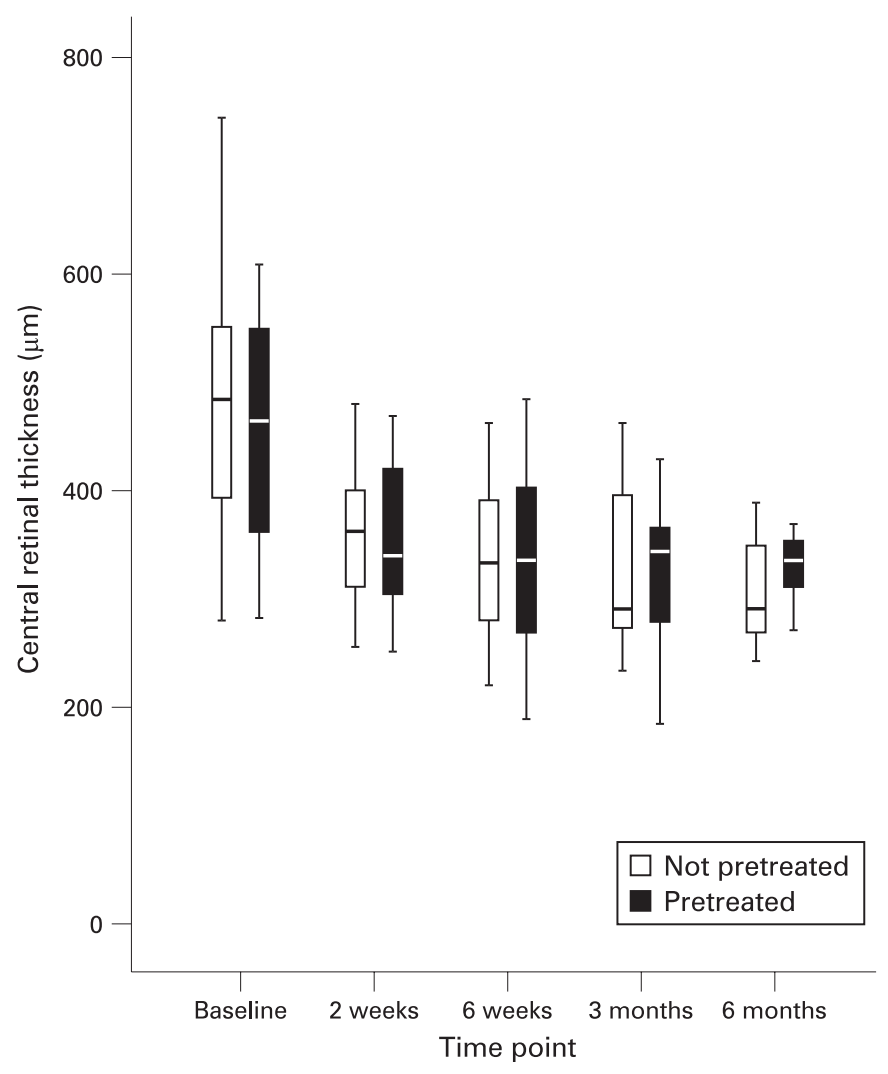

Figure 3 Comparison of central retinal thickness (measured by optical coherence tomography) between pretreated $(n=14)$ and non-pretreated $(n=20)$ cases of branch retinal vein occlusion. 
No side effects of the intravitreal injection of bevacizumab were seen.

\section{DISCUSSION}

Inhibitory drugs for the VEGF receptor have found their way into ophthalmology for the treatment of various diseases. The first to be introduced was bevacizumab (Avastin), a humanised monoclonal antibody that had previously been used for the treatment of metastatic colorectal cancer. Although it is an offlabel drug because of its low toxic side effects, its use in ophthalmology worldwide has shown an unprecedented increase. ${ }^{13}$ Previous studies have demonstrated its effectiveness in reducing macular oedema in eyes with retinal vein occlusions and age-related macular degeneration. ${ }^{514-16}$

This is the first large prospective clinical trial to demonstrate the effectiveness of intravitreal bevacizumab in reducing macular oedema. The OCT findings show a statistically significant reduction in central retinal thickness at all study time points. Moreover, mean visual acuity scores show significant improvement at all follow-up visits compared with baseline findings: a mean gain of three lines due to a mean increase of 15.3 letters on the ETDRS chart among all the patients.

One drawback of this study is the lack of testing of functional visual acuity, such as reading tests. In patients with focal macular oedema, reading problems are more pronounced than basic recognition of distantly presented optotypes. Evaluation of possible gains in reading performance is planned in a future study.

Subgroup analysis of early versus delayed treatment with intravitreal bevacizumab revealed no significant differences in visual acuity prognosis. This is in contrast with earlier findings of our group on treatment of macular oedema secondary to central retinal vein occlusion with intravitreal bevacizumab. ${ }^{16}$ In BRVO, unlike central retinal vein occlusion, perfusion of retinal structures can still be achieved by collaterals to the nonperfused area. This presumably results in less, or delayed, chance of irrevocable damage to the macula, with better prognosis for visual acuity even after delayed treatment of the oedema.

Although there is some controversy about its effectiveness, laser photocoagulation has been proposed to be the best treatment for persistent macular oedema following BRVO. ${ }^{17} 18$

In our study, five patients showed mild recurrent exudation after initially successful bevacizumab treatment. In these patients, complete resorption of macular oedema could be achieved with additional focal laser photocoagulation. Retinal thickness and visual acuity were stable for the whole followup period. Two of these cases were pretreated (with pars plana vitrectomy and peeling of the epiretinal membrane) and three were not. From these results, one could conclude that a combined treatment of bevacizumab and focal laser photocoagulation might be an even more effective approach. However, considering the non-pretreated cases, only two $(10 \%)$ of 20 patients in our trial needed additional laser treatment. Further studies will be necessary to determine whether this combined treatment would reduce the number of bevacizumab injections needed to resolve persistent oedema or if bevacizumab treatment can effectively reduce the necessity for grid laser photocoagulation in the long term.

Subgroup analysis revealed that pretreatment of BRVOs before bevacizumab injections resulted in a worse prognosis than when bevacizumab was the initial treatment. Barbazetto and Schmidt-Erfurth ${ }^{19}$ have shown that grid laser photocoagulation causes an increase in central scotoma size in patients with macular oedema after BRVO. Laser treatment led to decreased mean visual acuity scores in these eyes after 3 months. Analogously to that study, most pretreated eyes in our study had laser photocoagulation, which may explain the reduced visual acuity in this subgroup. In addition, the duration of symptoms was significantly longer in the pretreated cases than the non-pretreated cases: 53.7 (49) vs 28.7 (68) weeks $(p=0.001)$. The longer duration of macular oedema may also have contributed to the worse results for visual acuity. Although this may be true, even these eyes benefited from the intravitreal bevacizumab injections, on average, gaining 12.5 letters on the ETDRS chart.

In the Branch Vein Occlusion Study, eyes with macular oedema after BRVO either received grid laser photocoagulation or remained untreated. Eyes either received grid laser photocoagulation or remained untreated. After 3 years, $63 \%$ of eyes in the laser-treated group had gained two or more lines, whereas only $36 \%$ of untreated eyes reached that level. ${ }^{2}$ Bevacizumab treatment of BRVOs in the present study produced a similar outcome to the Branch Vein Occlusion Study: 62\% of patients gained two or more lines compared with prior treatment. However, an increase in visual acuity after bevacizumab treatment is observed after only a very short observation period and the effect of bevacizumab lasted for 6 months. Further studies are necessary to evaluate the long-term effect of antiVEGF therapy for the treatment of macular oedema secondary to BRVO.

In summary, we have shown that intravitreal injections of bevacizumab are an effective treatment option for eyes with macular oedema due to BRVO. Whether additional laser photocoagulation would produce additional benefit needs to be further evaluated. We can recommend this treatment as a primary approach for eyes with macular oedema secondary to BRVO.

\section{Competing interests: None declared.}

Part of this work has been presented at the 6th International Symposium on Ocular Pharmacology and Therapeutics (ISOPT), Berlin, 2006.

\section{REFERENCES}

1. The Eye Disease Case-control Study Group. Risk factors for branch retinal vein occlusion. Am J Ophthalmol 1993;116:286-96.

2. Argon laser photocoagulation for macular edema in branch vein occlusion The Branch Vein Occlusion Study Group. Am J Ophthalmol 1984;98:271-82.

3. Costa RA, Jorge R, Calucci D, et al. Intravitreal bevacizumab (Avastin) for central and hemicentral retinal vein occlusions: IBeVO study. Retina 2007:27:141-9.

4. Spaide RF, Fisher YL. Intravitreal bevacizumab (Avastin) treatment of proliferative diabetic retinopathy complicated by vitreous hemorrhage. Retina 2006;26:275-8.

5. Iturralde D, Spaide RF, Meyerle CB, et al. Intravitreal bevacizumab (Avastin) treatment of macular edema in central retinal vein occlusion: a short-term study. Retina 2006;26:279-84.

6. Vinores SA, Xiao WH, Aslam S, et al. Implication of the hypoxia response element of the VEGF promoter in mouse models of retinal and choroidal neovascularization, but not retinal vascular development. J Cell Physiol 2006;206:749-58.

7. Boyd SR, Zachary I, Chakravarthy U, et al. Correlation of increased vascular endothelial growth factor with neovascularization and permeability in ischemic central vein occlusion. Arch Ophthalmol 2002;120:1644-50.

8. Keck PJ, Hauser SD, Krivi G, et al. Vascular permeability factor, an endothelial cell mitogen related to PDGF. Science 1989;246:1309-12.

9. Leung DW, Cachianes G, Kuang WJ, et al. Vascular endothelial growth factor is a secreted angiogenic mitogen. Science 1989;246:1306-9.

10. Senger DR, Connolly DT, Van de Water $\mathrm{L}$, et al. Purification and $\mathrm{NH}_{2}$-terminal amino acid sequence of guinea pig tumor-secreted vascular permeability factor. Cancer Res 1990;50:1774-8.

11. Spandau U, Wickenhauser A, Rensch F, et al. Intravitreal bevacizumab for branch retinal vein occlusion. Acta Ophthalmol Scand 2007;85:118-19. 
12. Ferris FL 3rd, Kassoff A, Bresnick GH, et al. New visual acuity charts for clinical research. Am J Ophthalmol 1982;94:91-6.

13. Fung AE, Rosenfeld PJ, Reichel E. The International Intravitreal Bevacizumab Safety Survey: using the internet to assess drug safety worldwide. $\mathrm{Br} \mathrm{J}$ Ophthalmol 2006;90:1344-9

14. Rabena MD, Pieramici DJ, Castellarin AA, et al. Intravitreal bevacizumab (Avastin) in the treatment of macular edema secondary to branch retinal vein occlusion. Retina 2007; 27:419-25.

15. Pai SA, Shetty R, Vijayan PB, et al. Clinical, anatomic, and electrophysiologic evaluation following intravitreal bevacizumab for macular edema in retinal vein occlusion. Am J Ophthalmol 2007;143:601-6.
16. Priglinger SG, Wolf AH, Kreutzer TC, et al. Intravitreal bevacizumab for central retinal vein occlusion: six month results of a prospective trial. Retina 2007;8:1004-12.

17. Parodi MB, Spasse S, lacono $\mathrm{P}$, et al. Subthreshold grid laser treatment of macular edema secondary to branch retinal vein occlusion with micropulse infrared (810 nanometer) diode laser. Ophthalmology 2006;113:2237-42.

18. Battaglia Parodi M, Saviano S, et al. Grid laser treatment in macular branch retinal vein occlusion. Graefes Arch Clin Exp Ophthalmol 1999;237:1024-7.

19. Barbazetto IA, Schmidt-Erfurth UM. Evaluation of functional defects in branch retinal vein occlusion before and after laser treatment with scanning laser perimetry. Ophthalmology 2000;107:1089-98.

\section{BMJ Clinical Evidence - Call for contributors}

BMJ Clinical Evidence is a continuously updated evidence-based journal available worldwide on the internet which publishes commissioned systematic reviews. BMJ Clinical Evidence needs to recruit new contributors. Contributors are healthcare professionals or epidemiologists with experience in evidencebased medicine, with the ability to write in a concise and structured way and relevant clinical expertise.

Areas for which we are currently seeking contributors:

- Secondary prevention of ischaemic cardiac events

- Acute myocardial infarction

- MRSA (treatment)

- Bacterial conjunctivitis

However, we are always looking for contributors, so do not let this list discourage you.

Being a contributor involves:

- Selecting from a validated, screened search (performed by in-house Information Specialists) valid studies for inclusion.

- Documenting your decisions about which studies to include on an inclusion and exclusion form, which we will publish.

- Writing the text to a highly structured template (about 1500-3000 words), using evidence from the final studies chosen, within 8-10 weeks of receiving the literature search.

- Working with BMJ Clinical Evidence editors to ensure that the final text meets quality and style standards.

- Updating the text every 12 months using any new, sound evidence that becomes available. The BMJ Clinical Evidence in-house team will conduct the searches for contributors; your task is to filter out high quality studies and incorporate them into the existing text.

- To expand the review to include a new question about once every 12 months.

In return, contributors will see their work published in a highly-rewarded peer-reviewed international medical journal. They also receive a small honorarium for their efforts.

If you would like to become a contributor for BMJ Clinical Evidence or require more information about what this involves please send your contact details and a copy of your CV, clearly stating the clinical area you are interested in, to CECommissioning@bmjgroup.com.

\section{Call for peer reviewers}

$B M J$ Clinical Evidence also needs to recruit new peer reviewers specifically with an interest in the clinical areas stated above, and also others related to general practice. Peer reviewers are healthcare professionals or epidemiologists with experience in evidence-based medicine. As a peer reviewer you would be asked for your views on the clinical relevance, validity and accessibility of specific reviews within the journal, and their usefulness to the intended audience (international generalists and healthcare professionals, possibly with limited statistical knowledge).

Reviews are usually $1500-3000$ words in length and we would ask you to review between 2-5 systematic reviews per year. The peer review process takes place throughout the year, and our turnaround time for each review is 10-14 days. In return peer reviewers receive free access to BMJ Clinical Evidence for 3 months for each review.

If you are interested in becoming a peer reviewer for BMJ Clinical Evidence, please complete the peer review questionnaire at www.clinicalevidence.com/ceweb/contribute/peerreviewer.jsp 


\section{BJO}

\section{Intravitreal bevacizumab for the treatment of macular oedema secondary to branch retinal vein occlusion}

T C Kreutzer, C S Alge, A H Wolf, et al.

Br J Ophthalmol 2008 92: 351-355 originally published online January 22, 2008

doi: 10.1136/bjo.2007.123513

Updated information and services can be found at:

http://bjo.bmj.com/content/92/3/351.full.html

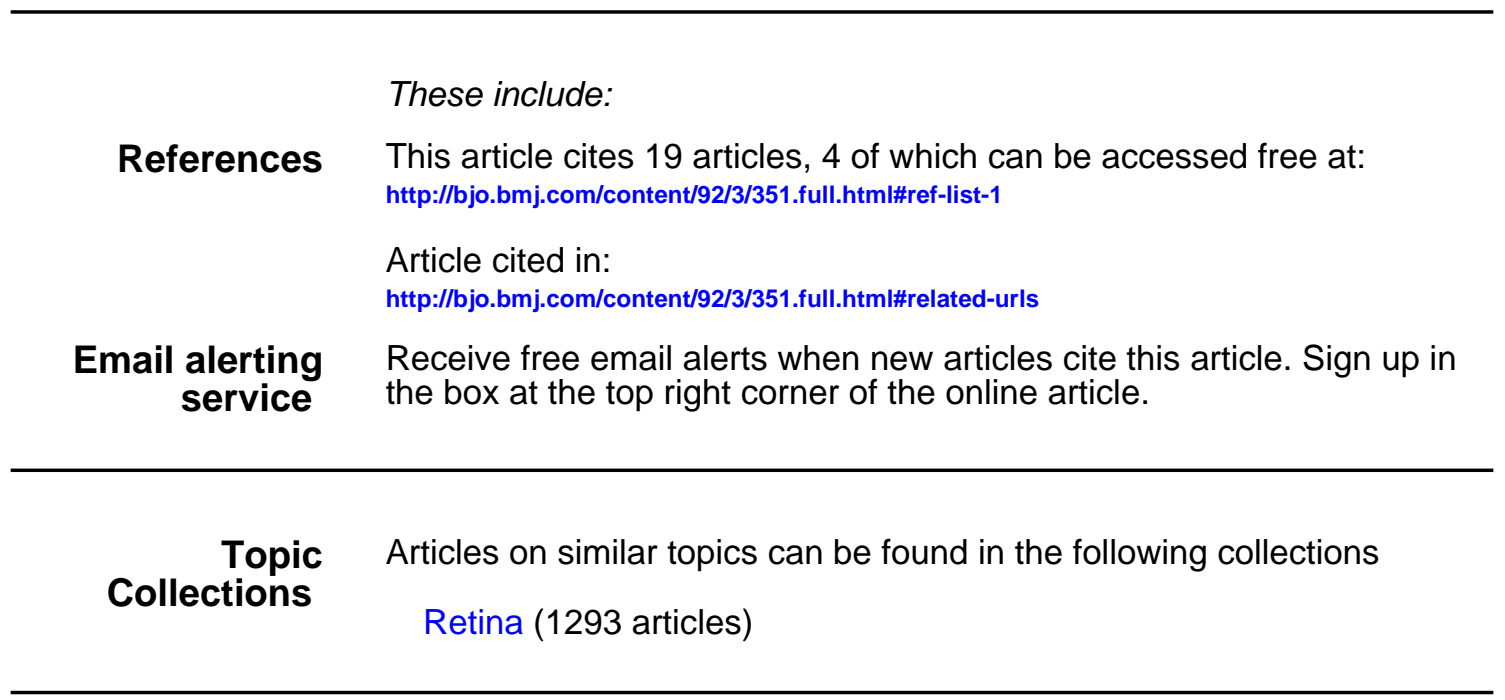

Notes

To request permissions go to:

http://group.bmj.com/group/rights-licensing/permissions

To order reprints go to:

http://journals.bmj.com/cgi/reprintform

To subscribe to BMJ go to:

http://group.bmj.com/subscribe/ 